Luca Basso. Titular de filosofía política del Dipartimento di Scienze Politiche, Giuridiche e Studi Internazionali, Università degli Studi di Padova. Forma parte del comité científico de la Escuela de especialización en filosofía de la misma universidad y del comité editorial de revistas académicas como Storia del pensiero politico y Consecutio rerum. Sus ámbitos de investigación conciernen a la filosofía del primer milenio, la teoría política alemana y francesa de los siglos XVIII y XIX, el marxismo, la teoría crítica y la filosofía contemporánea francesa. Dentro de sus publicaciones recuérdense: Individuo e comunità nella filosofia politica di Leibniz (2005), Società e isolamento: la singolarità in Marx (2008, trad. ingl. 2012), Republic and Common Good in Leibniz' Political Thought, en "Studia Leibnitiana" (2011), Agire in comune. Antropologia e politica nell'ultimo Marx (2012, trad. ingl. 2015), Inventare il nuovo. Storia e politica in Jean-Paul Sartre (2016), Marx: la produzione del soggetto (2018, eds. Michele Basso, Fabio Raimondi y Stefano Visentin), Leibniz und das Naturrecht (2019).

Contacto: luca.basso@unipd.it 


\title{
IN THE CRITQUE OF
}

\section{POLITICAL ECONOMY AND POLITICS ${ }^{1}$}

\author{
Luca Basso \\ Università degli Studi di Padova
}

\section{Abstract}

This essay is centered on the element of the class, often removed in these years, focusing on its conceptual status, its relationship with reality and its critical potential. To address this theoretical plexus, the reference to Marx remains crucial. My essay, which tries to make reflection of Marx (and some Marxist theorists) interact with the contemporary debate, is divided into three parts. The first deals with the relationship between the critique of political economy and politics. The second focuses on the consequences of this approach on the status of the class. In the third part I try to highlight strengths and open problems in the investigated approach. What is at stake is to understand how we can relaunch this question in today's scenario, changed with respect to the Marxian context, and on the basis of an interaction between the lines of class and race.

\section{Keywords}

Class, Marxism, critique of political economy, politics, race.

\section{Resumen}

Este ensayo se centra en el elemento de la clase, a menudo desatendido en estos años, en su significado conceptual, su relación con la realidad y su potencial crítico. Para

1. Fecha de recepción: 20 de febrero 2019; fecha de aceptación: 4 de marzo 2019. El presente artículo es resultado de un proyecto de investigación desarrollado con el Dipartimento di Scienze Politiche, Giuridiche e Studi Internazionali, Università degli Studi di Padova. 
afrontar este conjunto teórico es fundamental remitir a Marx. Al intentar reflexionar sobre cómo Marx (y algunos teóricos marxistas) intenta interactuar con el debate contemporáneo, el ensayo se divide en tres partes. La primera atañe a la relación entre la crítica de la economía política y la política. La segunda se fundamenta en las secuelas de este enfoque en el estatuto de clase. En la tercera se intenta hacer el balance de la situación y dar paso a nuevos enfoques de investigación. El objetivo es entender cómo promover esta cuestión en el escenario actual, diferente del contexto marxiano, partiendo de una interacción entre clase y raza.

\section{Palabras clave}

Clase, Marxismo, crítica de la economía política, política, raza. 
Este ensayo se centra en el elemento de la clase, a menudo desatendido en los últimos años, haciendo hincapié en su estatuto teórico, su relación con la realidad y sus potencialidades críticas. Las mismas cuestiones de género y raza, al cruzarlas -no de forma simple e inmediata, sino problematizándolas- con la dimensión de la clase adquieren otro significado respecto de si se interpretan a partir de una lógica abstracta de los derechos humanos, de la dignidad de todo individuo, etc. Para afrontar este conjunto teórico, considerando los dos polos del título -crítica de la economía política y política- es fundamental remitir a Marx. El pensador alemán -el cual, tras finalizar el socialismo real y por un cierto conformismo cultural, fue olvidado o unilateralmente criticado- en la actualidad es muy estudiado a nivel internacional, sobre todo en países sin tradición marxista. De ahí que hayan brotado nuevas opiniones que no han puesto en tela de juicio, pero sí problematizado, algunas de las tesis clásicas de los marxismos. Se remite a Marx porque hay la convicción de que existe un análisis crítico insuperado del capitalismo, no por esto exento de problemas. Además, de Marx a hoy ha habido muchos cambios en el desarrollo del capitalismo. Este ensayo -que intenta poner en relación la reflexión marxiana con el debate contemporáneose divide en tres partes. La primera tematiza la relación entre crítica de la economía política y política. La segunda profundiza en las secuelas de este enfoque en el estatuto de clase. La tercera parte intenta arrojar luz sobre los puntos fuertes y los problemas pendientes de la propuesta.

\section{Teoría y práctica}

Antes de interrogarnos acerca de la relación entre crítica de la economía política y política en Marx (y Engels), se necesita hacer algunas premisas. Primero, el elemento de la crítica parece constituir realmente el discurso marxiano, y prueba de ello es que todos los títulos y subtítulos de las obras principales remiten a la "crítica": Una contribución a la crítica de la economía política, Líneas fundamentales de la crítica de la economía política, El capital. Crítica de la economía política... Cuando manifiesta su dispositivo teórico, Marx emplea a menudo la voz crítica, y no tanto el término ciencia, aunque sin alejarse de una exigencia de cientificidad. Hay que añadir otro problema. A pesar de seguir los pasos tradicionalmente reconocidos, Marx esboza una crítica de la política antes de elaborar una crítica de la economía política. Las primeras obras marxianas se caracterizan por criticar la política moderna y obviamente es una crítica inmanente, que no añora las 
épocas anteriores y las formas precapitalistas. Solo luego Marx elaborará una crítica de la economía política, antes de forma solo esbozada y luego sistemática.

En general, la relación entre las dimensiones en cuestión es articulada, irreducible a una derivación linear. Louis Althusser -sobre todo en Lire le Capital- destaca que Marx, aunque se queda en el grupo de los economistas políticos por lo que al lenguaje, a la terminología y a los discursos se refiere, con respecto a estos realiza no solo un desplazamiento, sino también una desestructuración. Una "revolución teórica" similar no se asemeja a ninguna de las "revoluciones teóricas" que se sucedieron en la historia del pensamiento, ya que se apropia de forma totalmente inédita de la dimensión práctica. A partir de aquí, Althusser estudia los rasgos distintivos del materialismo marxiano, afimando que el eje de la tesis de Marx concierne al principio de distinción entre lo real y el pensamiento. Una cosa es lo real con sus aspectos diferentes y otra cosa es el pensamiento de lo real con sus aspectos diferentes. Este principio de distinción - para Althusser - implica dos tesis fundamentales: la primera es la tesis materialista de la primacía de lo real sobre su pensamiento, porque el pensamiento de lo real presupone la existencia de lo real independientemente de su pensamiento; la segunda es la tesis materialista de la especificidad del pensamiento y del proceso de pensamiento hacia lo real y el proceso real (Althusser, 2006, pp. 176-177). Del discurso marxiano emergen dos elementos clave. El primero es el reconocimiento de la prioridad de la realidad, de la "verdad efectual de la cosa" -recordando la extraordinaria expresión de Maquiavelo- en su singularidad, respecto del pensamiento (Althusser, 1995). Lo chocante es que el de Marx no es solo un análisis ubicado en lo real, sino también una tensión para su transformación: de ahí que la referencia a lo real llegue a adquirir un alcance revolucionario. En Les formes de l'histoire y sobre la base de un enfoque profundamente diferente del de Althusser, Claude Lefort insiste en la existencia de una pasión realista común a Maquiavelo y Marx. El realismo se fundamenta en la idea de que la realidad empírica, compuesta por la historia de los hombres, puede llegar al conocimiento, de ahí que sea decisiva para articular una acción adecuada. En Marx, el aspecto en apariencia paradójico es que, por un lado, lo real es lo que es y, por otro lado, de esta posición se consigue una tarea práctica, un proceso de realización, un entusiasmo dirigido a devolver a lo real su verdadera identidad. En todo caso, más allá de su crítica al socialismo utópico, no creemos que la aceptación de la "verdad efectual de la cosa" pueda sic et simpliciter contraponerse a la utopia. Lefort subraya que, en su forma realizada, la realidad es la política (Lefort, 2005, p. 192). Este cruce, en apariencia paradójico, entre la adhesión a la "verdad efectual de 
la cosa" y la tensión hacia la transformación no adquiere un carácter genérico, sino que se inserta en la acción concreta, potencialmente eficaz de fuerzas sociales en un contexto específico.

Regresando a Althusser, además de la primera tesis - que consiste en la prioridad de la realidad sobre el pensamento - también es importante la segunda tesis marxiana, que destaca la autonomía del pensamiento respecto de la realidad. Está claro que solo se trata de una independencia parcial, en el sentido de que, de lo contrario, faltaría toda relación entre las dos tesis. De todos modos, si no existiera esta autonomía parcial, ya no tendría sentido el proyecto mismo de la crítica de la economía política. Marxianamente, no se puede crear una práctica política productiva sin teoría. Si se nos quedara anclados a la coyuntura en su especificidad y en su carácter metamórfico desde los puntos de vista espacial y temporal, sería imposible crear una articulación global del discurso relativo a la manera de producción capitalista. En efecto, es inevitable que la crítica de la economía política presente un elemento de abstracción, no considerado en términos restrictivos. Este enfoque presupone otro aspecto importante, o sea la crítica a la homología hegeliana entre realidad y pensamiento: se nos halla frente al reconocimiento materialista de la no-coincidencia entre objeto real y objeto del conocimiento. En todo caso, la crítica de la economía política no se configura como un elemento que sirve simplemente para la práctica: no se hace una mera deducción de un plan a partir del otro, ni la práctica procede inmediatamente de la teoría. Se consigue que la misma relación entre esas dimensiones es, por un lado, apremiante y con "carga" revolucionaria y, por otro lado, irreducible a soluciones predeterminadas. Por lo general, el planteamiento de Althusser corre el riesgo de alejar la crítica de la economía política de la política: en una visión fundamentada en un "proceso sin sujeto", es difícil articular los espacios de los sujetos, a no ser de manera puramente "intersticial". A pesar de sus límites, la exploración de la posición althusseriana nos ha proporcionado la posibilidad de hacer emerger la relación no linear entre crítica de la economía política y política.

\section{De las clases a las luchas de clase. Entre estructura y conyuntura}

La segunda parte del ensayo analiza las consecuencias de la relación presente en el estatuto de la clase, partiendo de la observación de una (real o aparente) contradicción entre su lectura económica y política. Una interpretación problematizante de la cuestión se halla en el libro de Etienne Balibar e Immanuel Wallerstein, Race nation classe. 
Les identités ambigues (Balibar \& Wallerstein, 1988, sobre todo pp. 207-246). Lo problemático e interesante al mismo tiempo es que no hay una inmediata cohesión entre los escritos de crítica de la economía política -con su carácter de abstracción- y las obras histórico-políticas -con su inserción en una coyuntura determinada. El estatuto de la clase se mueve entre dimensión económica y dimensión política del discurso. Prima facie parece que de la crítica de la economía política emerge una estructura dual -clase obrera y clase de los capitalistas- en la que la clase obrera sirve para el trabajo, mientras que la capitalista para el capital, sobre la base de una desustancialización radical. Esto es el lado económico de la clase. Además, las clases presentes en los escritos políticos a menudo no son dos: si se analiza La lucha de clases en Francia y El 18 de Brumario, en la "verdad efectual de la cosa" las clases son más de dos. Piénsese en la presencia de los aristócratas, de los campesinos, de los lumpenproletarios, en el hecho de que los burgueses se dividen en su interior (gran burguesía y pequeña burguesía o - si empleamos una categorización diferente - burguesía financiera y burguesía industrial). A menudo las clases se fotografian en un momento de insurrección revolucionaria, con la función expansiva del proletariado. Ahí va el lado político de la clase.

Solo sobre la base de aquella abstracción conceptual típica de la crítica de la economía política se puede enfocar el interés por dos clases, y no se puede hacer desde siempre y para siempre, sino en el interior de un horizonte históricamente determinado, aquel del modo de producción capitalista (del cual, en el Capital, sobre todo en el capítulo sobre la acumulación originaria, Marx intenta trazar una genealogía, mejor dicho diferentes genealogías, haciendo hincapié en aquellos cambios que "hicieron historia"), en su diferencia específica respecto de las formas productivas anteriores. A pesar de algunas oscilaciones en Marx (por ejemplo, en el Manifiesto se describe toda la historia como una historia de luchas entre las clases. Cfr. Vincent, 2001, pp. 82-88), de forma real las clases solo se dan en el interior del sistema capitalista, al historicizarse y despersonalizarse las relaciones de producción. Es más, se puede hablar de clases stricto sensu en la situación específica del capitalismo industrial, y en el Capital con referencia específica a Inglaterra, es decir, el país en el que el desarrollo capitalista y la polarización en clases se manifestaron más claramente. La reflexión marxiana sobre la clase no se puede comprender sin considerar, por un lado, la revolución industrial y, por el otro, la revolución francesa (Bongiovanni, 1989): con Marx se asiste a una radical historicización (que no significa historicismo) de las categorías, sobre la base de una continua interacción entre materialismo e historia (Basso, 2018). De ahí que sea crucial la fase 1789-1848, es decir, aquel pasaje de la revolución política a la revolución social en el que insistieron 
muchos estudiosos: piénsese, por ejemplo, en las obras de Reinhart Koselleck, en particular Vergangene Zukunft (Koselleck, 1979, pp. 67-86). Tanto el discurso de los economistas políticos clásicos como aquel de Sieyès son fundamentales para comprender las premisas de la clase como clase social. Por otro lado, el mismo Marx reconoce haber sacado el concepto de lucha de clase de los historiadores de la Restauración, esto es, de un escenario, aunque reactivo, totalmente interno a la Revolución francesa. También cabe añadir que estamos ante una disimetría entre clase obrera y clase de los capitalistas. Podría afirmarse que la única clase verdadera, en El capital, es la clase obrera. La clase de los capitalistas (es significativo que Marx hable a menudo de clase de los capitalistas, en lugar de clase capitalista) representa una unión siempre inestable entre las diferentes fracciones de los capitalistas (de tipo industrial, comercial, financiero, etc.), concurrentes entre sí, cuya unión ficticia queda sellada por el Estado. La clase de los capitalistas es lo que es en el Estado y con el Estado.

El horizonte trazado puede complicarse aún más. Tal y como se ha puesto de manifiesto antes, no se da una inmediata cohesión entre crítica de la economía política y política, puesto que esta última no puede considerarse, de manera intelectualista, como derivada de una teoría prencostituida, sino que se inserta en la coyuntura. Todo el pensamiento político marxiano se puede interpretar como un "pensar en la conyuntura", con su carácter metamórfico. Y la reflexión siguiente a la derrota de 1848 se puede leer como una especie de rectificación del Manifiesto del partido comunista: no una autocrítica, sino una rearticulación del discurso a partir de un cuadro social y político que se revela diferente (Balibar, 1974, pp. 65-102). Sin embargo, este enfoque no lleva a una dicotomía entre la clase como elemento económico - en la crítica de la economía política - y la clase como elemento político - en los escritos histórico-políticos -. En este sentido es particulamente icástico un pasaje del Pos-escrito a la segunda edición del Capital: "Leconomia politica, in quanto è borghese, cioè in quanto concepisce lordinamento capitalistico, invece che come grado di svolgimento storicamente transitorio, addirittura all'inverso come forma assoluta e definitiva della produzione sociale, può rimanere scienza (Wissenschaft) soltanto finché la lotta delle classi (Klassenkampf) rimane latente o si manifesta soltanto in fenomeni isolati” (Marx, 1991, pp. 19-20, trad. it., pp. 38-39). Lo chocante es la relación inseparable entre la crítica elaborada por el teórico y la práctica política del militante, pero sin la idea de que la práctica política represente la mera ejecución de la crítica elaborada por el teórico. Es un elemento extrateórico -y directa y violentemente político como la lucha de clase- el que hace "estallar" la economía política clásica y que hace entrar en crisis la cientificidad. 
Por un lado, cabe considerar el hecho de que la política no se deduce directamente de la crítica de la economía política y, por el otro, el carácter político de esta última. Tal y como ha subrayado resueltamente el obrerismo, por ejemplo, Tronti (1980), emerge el alcance intrínsecamente político de la clase que, al mismo tiempo, exige una radical refundación de la política oficial: en todo caso, las clases se manifiestan claramente en la lucha de clase. En todas las obras marxianas se difunde la idea de que los individuos forman una clase cuando luchan contra otra clase. Sin embargo, a pesar de insertarse en el escenario económico, social y político de la dialéctica capital-trabajo, esta idea no puede hipostasiarse ni sociológica ni filosóficamente. Es un concepto político, pero basado en un dato económico, y no puede entenderse solo a través de una racionalidad instrumental. La clase no es solo razón, sino también pasión, sentido de pertenencia, forma de vida. Además, sería interesante hacer interactuar el aspecto del que se acaba de hablar con la que Edward P. Thompson llama "la economía moral", sobre la base de una porosidad entre elementos obreros en sentido estrecho y elementos populares (Thompson, 1963). El estatuto de la clase es -por así decirlo- impuro, irreducible a una codificación sistemática. Además, tal y como se destaca muy eficazmente en $\mathrm{El} 18$ de Brumario, se cruzan una lectura de las condiciones socioeconómicas y un análisis del imaginario social que, sin embargo, produce consecuencias materiales. A este respecto, es muy interesante el último breve capítulo, nunca acabado, sobre las clases sociales en el tercer libro del Capital (Marx, 1989, pp. 892-893, trad. it., pp. 1003-1004). Las clases no son dos, sino tres, obreros asalariados, capitalistas y terratenientes. Marx se pregunta qué es lo que construye a una clase social, y afirma que, contrariamente a lo que podría opinarse, no la define la renta. Esto se debe a que la clase no puede reducirse al dato económico y perfilarse una vez por todas. La interrupción del manuscrito también revela un estancamiento a la hora de individualizar sus signos distintivos. Tal y como emerge también de las reflexiones de un economista agudo como Joseph Schumpeter, para Marx el concepto de clase era tan ínsito en su investigación como para parecerle obvio, no representaba un problema teórico, lo daba por descontado (Tronti, 2008).

No se va a tratar aquí la compleja cuestión de la teoría de la revolución y del papel de la dictadura del proletariado en la llamada fase de transición (Hobsbawm, 1978, pp. 256-257), que llevó a numerosos problemas teóricos y políticos que, sin embargo, deben entenderse en la justa medida. Se nos limita a subrayar que el lema "dictadura", en la época marxiana, tenía un significado profundamente diferente del que adquiriría en el siglo XX. A pesar de las posibles variaciones, a menudo la lucha entre las clases no se configura como una guerra civil (para emplear un concepto empleado a menudo 
por Marx). Desde este punto de vista, es verdad que el sistema capitalista representa la primera manera de producción con un carácter antagónico, materializado en clases contrapuestas, pero este antagonismo resulta ser potencial, a menudo no in actu. Sigue siendo importante la distinción - que no se debe entender de manera linear sobre la base de una sucesión tempora l- entre clase en sí, comunidad objetiva de condiciones, y clase por sí, organizada políticamente. No se pasa automáticamente del primer elemento al segundo, y en el panorama actual el problema en cuestión emerge con mayor dramaticidad.

De ahí que regrese con toda su importancia (y también ambivalencia) el análisis del papel de la política (Maguire 1978; Gilbert 1981; Barbier 1992; Texier, 1998). Marx critica a esta última, a veces con el riesgo de considerarla en términos solo críticos y destructivos. Pero se ha preferido poner en el título del ensayo el lema "política" en lugar de "crítica de la política", puesto que, aunque con problemas internos, emerge la importancia de la política en sus escritos históricos, del 48 a la Internacional, a la Comuna, y también en El Capital, por ejemplo, en las luchas para la reducción de la jornada laboral. En lo que se refiere a estas últimas, no se puede sino recordar el logro de una "ley de Estado" que, frente al pomposo catálogo de los derechos inalienables del hombre, se configura como una modesta Magna Charta de una jornada laboral limitada por la ley (Marx, 1991, p. 320, trad. it., pp. 338-339). La lucha de clase, con su carácter político, no puede eximirse de cruzar críticamente (pero sobre la base de una crítica inmanente, no de un absoluto "más allá"), la dimensión jurídica. En todo caso, contra Bakunin Marx subraya que la clase obrera debe interesarse por la política. En una carta de 1870 a Paul y Laura Lafargue, Marx afirma que "ogni movimento di classe in quanto movimento di classe è ed è sempre stato necessariamente un movimento politico" (Marx, 1975, p. 675, trad. it., p. 728). En Sobre la acción política de la clase obrera, Engels subraya: "Il partito operaio come partito politico esiste e vuole agire politicamente, e predicare la sua astensione significa mandare in rovina l'Internazionale [...]. Dopo la Comune, che ha posto all'ordine del giorno l'azione politica dei lavoratori, l'astensione è impossibile [...]" (Engels, 2008, pp. 412-413, trad. it., pp. 397-398).

Un problema que sigue pendiente es la relación entre político y estatual. Muy significativo a este propósito es el curso sobre el Estado de Pierre Bourdieu de 1989-1990 en el Collège de France (Bourdieu, 2012): el Estado no es un bloque - tal y como a veces Marx parece afirmar - sino un conjunto de fuerzas. Aquí no nos vamos a preguntar si existe o no existe una teoría del Estado en Marx: piénsese en el debate entre Norberto Bobbio y Antonio Negri en Italia, y en las discusiones de Louis Althusser y Nicos Poulantzas en 
Francia (Bobbio, 1976; Negri, 2006; Althusser, 1995; Poulantzas, 1964). Se nos limita a subrayar que la definición de Estado, presente en el primer libro del Capital, como violencia (Gewalt) concentrada y organizada (Marx, 1995, p. 779, trad. it., p. 814) es todo menos que incompatible con la definición realística de Estado proporcionada por Max Weber. Por lo demás, "diritto contro diritto. Tra diritti uguali decide la forza (Gewalt)" (Marx, 1995, p. 249, trad. it., p. 269). En la modernidad se enfrenta un nexo estructural entre Staat y Gewalt, tal y como ponen de manifiesto los léxicos de los conceptos políticos (piénsese sobre todo en el Lexikon al cuidado de Brunner, Koselleck y Conze: Faber, Ilting \& Meier, 1982). De todas formas, de las primeras a las últimas obras, Marx concibe el comunismo como muy lejano de lo que las experiencias históricas del siglo XX del "socialismo real" producirían, esto es, sobre la base de una crítica radical al "monstruo frío" del Estado y de la tentativa de articular una forma política expansiva más allá de la sociedad civil y del Estado, las dos caras de la misma moneda en la modernidad política (Basso 2008; Basso 2012).

\section{Problemas abiertos}

Al querer explicitar mejor algunos aspectos significativos y algunas cuestiones parcialmente no resueltas, se nos detiene en las implicaciones de la disertación sobre el "estado de cosas presente" y su crítica. Un primer problema atañe a la composición de clase. Aunque Marx hable política y dinámicamente de la clase, y aunque en las obras de los años sesenta y setenta tome cada vez más en consideración situaciones extraeuropeas o externas a la Europa occidental (por ejemplo, Rusia), no hay duda de que, en El Capital, él ha individualizado su perspectiva de análisis en el capital procedente de la revolución industrial en Europa, con énfasis en la gran industria inglesa. En el panorama actual se nos enfrenta a una situación muy diferente, en la que la clase obrera no ha desaparecido, sobre todo si lo pensamos en términos de economía-mundo (piénsese en la creciente proletarización en China: Arrighi \& Silver, 1999), pero seguramente ha habido cambios muy fuertes respecto de la época marxiana. Tal y como se destaca de diferentes estudios (por ejemplo, Sassen, 1998; Sassen, 2006; Sennett, 1998; Harvey, 2010), la crisis del Welfare State - y, por ende, de las estrategias político-económicas de derivación keynesiana - ha intensificado los mecanismos de competencia, que obviamente necesitan del papel del Estado, pero sobre la base de una modalidad parcialmente diferente con respecto al pasado, aunque el Estado sigue siendo el garante final de los derechos de propiedad. 
En la actualidad, la jornada laboral está formada por un mosaico de personas que operan con tiempos diferentes y cada vez más individualizados, mientras la empresa ejerce un control aún más minucioso que en el pasado. Además, la situación actual se caracteriza por una creciente financiarización de la acumulación del capital, a la que se añade - por lo general - una progresiva polarización en la distribución de la riqueza. Esta dinámica hace que el pasaje de la clase en sí a la clase por sí sea cada vez más complicado. Sin embargo, no hay que olvidar que, aunque en un escenario histórico diferente, en Marx - en particular en El 18 de Brumario, había destacado la idea de que, sobre todo en los períodos de crisis, las clases como grupos sociales descritos por intereses precisos y susceptibles de una representación política directa pueden descomponerse.

El segundo problema, muy enlazado con el estatuto de clase, es la relación entre clase, movimiento y partido políticos. Si, por un lado, Marx intenta anexar, aunque inestablemente, estos elementos, por otro lado, tras los dramáticos desarrollos del "siglo breve" y el fin del socialismo real se ha realizado -aunque de forma diferente en los diversos países- el asolamiento de las formas organizadas, políticas y sindicales. Lejos de deducir la práctica de una teoría preconstituida, la descomposición de las estructuras organizadas -cada vez más cosificadas - y la fragmentación individualista convierten en cada vez más complicada la porosidad entre crítica y acción política concreta.

El tercer problema es la manera de producción capitalista que, en Marx, presenta un carácter competencial y aún no monopolista. Un análisis histórico-económico, que quisiera estudiar los cambios en la configuración del capitalismo, haría emerger que, tras el período marxiano, hacia finales del siglo XIX y sobre todo con la primera guerra mundial, el sistema capitalista se ha caracterizado cada vez más en términos administrativos y burocráticos, y la sociedad burguesa -con sus rasgos expansivos también- ha sufrido mucho por un proceso de fraccionamiento con resultados asoladores. Después de esa fase y hasta la actualidad, los procesos de financiarización y privatización han vuelto a articular y complicar más el cuadro.

El cuarto problema, que atañe tanto a un elemento estructural del discurso marxiano, como a una cuestión que sigue actual a pesar de los cambios habidos, es la relación entre lo estatual y lo global. A este respecto, cabe recordar unas consideraciones de Cesare Luporini para el cual, por su naturaleza, la manera de producción capitalista desde el principio se desarrolla con una doble finalidad: la primera es la creación de un mercado interno de dimensiones considerables (por esto mismo la burguesía llega a ser clase dominante y dirigente sobre bases generalmente 'nacionales' y unificadas estatualmente), mientras que la segunda es el 'sistema global' (o sea la creación del 
mercado mundial) en el cual se produce la competencia de las diferentes burguesías nacionales y el desarrollo desigual de los diversos países afectados por el desarrollo capitalista. Para Luporini, todo esto se halla empíricamente en El Capital de Marx, menos la conceptualización de estos dos lados simultáneos, es decir, la demostración de su relación necesaria y reciprocidad (Luporini, 1978, p. 49). Este problema - del que Marx se había enterado sin sistematizarlo - de la relación entre dimensión estatual y global se repercute mucho en la noción de clase que, por un lado, se enlaza con situaciones estatuales sobre la base de una diversificación extrema y, por otro lado, se inserta en un sistema capitalista dirigido estructuralmente al mercado mundial y, por ende, con carácter global.

Para afrontar estas cuestiones sería útil considerar la clase de manera que se deje abierta, dinámicamente, la relación entre crítica de la economía política y política en un cuadro que ha cambiado y que, por ende, necesita de continuas rectificaciones. Opinamos que sigue siendo crucial la tesis de que, a diferencia de los socialistas, Marx piensa que el capital no es una cosa, sino una relación social, de ahí que una crítica a este deba tener en consideración constantemente ese elemento. Además, no se puede olvidar que la fuerza de trabajo, incorporada en el proceso de producción, se concibe como "l'insieme delle attitudini fisiche e intellettuali che esistono nella corporeità, ossia nella personalità vivente di un uomo [...]" (Marx, 1995, p. 181, trad. it., p. 200. Cfr. Vadée, 1992, pp. 283 y ss.). Nos parece fundamental que se hable de actitudes no solo físicas sino también intelectuales. La relación entre fuerza de trabajo y trabajo puede parangonarse al nexo entre lo que es en potencia y lo que es en acto. Cuando se vende algo que solo existe en la corporeidad, no puede separarse de la singularidad viviente del trabajador. Emerge la ambivalencia de la fuerza de trabajo que, por un lado, sirve para valorizar el capital y, por otro lado, puede tener otros desarrollos sobre la base de una oposición a esta lógica. De todos modos, la explotación representa un proceso de dos caras, caracterizadas por los términos - recíprocos - de plustrabajo y plusvalía. El plustrabajo representa la organización "concreta" del derroche de fuerza de trabajo social, y la plusvalía se configura como el movimiento "abstracto" del valor, que Marx llama "automovimiento" del capital (Balibar, 1997, p. 238). Este es el efecto de una relación social en la que la fuerza de trabajo se considera como mercancía, de ahí que presuponga una serie de condiciones inestables a crear en la esfera de la producción o fuera de esta, en el espacio social mantenido por el Estado, esto es, un Estado nunca completamente autónomo. Nunca ha existido una explotación absolutamente pura, es decir, desvinculada de un dominio político. Las condiciones a las que se nos enfrenta son sobre todo 
políticas, o bien dependen de un cruce continuo de lo económico con lo político. En este proceso de trabajo capitalista, para Marx la fuerza de trabajo no es solo mercancía, sino también división entre trabajo manual y trabajo intelectual, combinación jerárquica de trabajo cualificado y de trabajo no cualificado, empleo de mujeres, atracción y repulsión de mano de obra inmigrada.

En lo que se refiere al elemento que se acaba de subrayar, el sistema capitalista elabora una jerarquización en la cual se comprometen también a las mujeres, mientras la fuerza de trabajo migrante desempeña una función subalterna. Aquí no se quiere hallar en Marx ya una plena conciencia del cruce decisivo entre género, clase y raza: desde algunos puntos de vista, Marx sigue pendiente del contexto del siglo XIX, con todos sus límites posibles. Por lo que al racismo se refiere, no se trata de considerar a Marx como un "poscolonial" ante litteram, sino de destacar que en él aumenta la conciencia de la recíproca interacción entre dinámicas clasistas y dinámicas racistas, esto es, de la necesidad de una crítica capaz de atravesar esta relación (Balibar \& Wallerstein, 1988). Muchas reflexiones de las últimas décadas han intentado profundizar en este análisis. Si no se quiere interpretar el fenómeno contemporáneo del racismo en términos culturalistas, crremos que es más provechoso rearticular la cuestión de la clase sobre la base del cruce que nos ocupa. Hoy en día la centralidad del conjunto Estado-territorio-pueblo va disminuyendo, así como se van creando nuevos confines, reales o imaginarios (pero de todos modos portadores de efectos materiales), sobre la base de procesos que son al mismo tiempo de desterritorialización y deterritorialización (por lo demás, desde hace más de un siglo se habla de crisis del Estado y hay como la impresión de que se ha declarado demasiado pronto su muerte...).

Este escenario remite a la cuestión de las migraciones. De hecho, el primer libro de El Capital cierra refiriéndose precisamente a las migraciones. El capitalismo mundial produce nuevos confines y los migrantes representan -por así decirlo- las nuevas "clases peligrosas": desde este punto de vista, se abre un conflicto social con nuevas líneas de fractura, aunque este elemento no elimina la lucha en los caminos tradicionales. En este panorama global se hace relevante la subjetividad de los migrantes, en su irreducibilidad a la dimensión de la estatualidad y en su confrontación (que a menudo es un choque en términos dramáticos) con las dinámicas estatuales. Van a desmembrarse las categorías y las estrategias políticas, en las que van a entrecruzarse cada vez más sujetos "trabajadores", "interinos" y "migrantes". Ya que el horizonte actual es muy fragmentado desde el punto de vista de las condiciones laborales, no se trata de haber individualizado una nueva clase revolucionaria, proporcionando una respuesta política inmediata, sino 
de destacar que los migrantes se configuran como uno de los indicadores de los cambios ocurridos. A pesar del diferente cuadro social y político, sigue importante la afirmación de El Capitale sobre la relación entre el fin de la segregación racial y emancipación: "Negli Stati Uniti dell'America del Nord ogni movimento operaio indipendente rimase paralizzato, finché la schiavitù deturpava una parte della repubblica. Il lavoro in pelle bianca non può emanciparsi in un paese dove viene marchiato a fuoco quand’è in pelle nera" (Marx, 1995, p. 318, trad. it., p. 337).

Traducción del italiano de M. Colucciello

\section{Referencias}

[Para indicar la edición alemana K. Marx \& F. Engels. Werke. Berlin: DietzVerlag, empleo la abreviatura $M E W]$

Althusser, L. (1995). Machiavel et nous (1972-1986). En L. Althusser, Écrits philosophiques et politiques (pp. 42-168). II. Paris: Stock/Imec.

Althusser, L. (1995). Sur la reproduction. Puf: Paris.

Althusser, L. (2006). L'oggetto del "Capitale”. En VV.AA., Leggere il Capitale (1965, pp. 1-185). trad. it. Milano: Mimesis.

Balibar, É. (1974). Cinqétudes de matérialismehistorique. Paris: François Maspero.

Balibar, É. \& Wallerstein, I. (1988). Race nation classe. Les identités ambiguës. Paris: La Découverte.

Balibar, É. (1997). La crainte des masses. Paris: Galilée.

Barbier, M. (1992). La pensée politique de Marx. Paris: L'Harmattan.

Basso, L. (2008). Socialità e isolamento: la singolarità in Marx. Roma: Carocci.

Basso, L. (2012). Agire in comune. Antropologia e politica nell'ultimo Marx. Verona: Ombre corte.

Basso, L. (2018). Materialismo e storia. Dall' "Ideologia tedesca" alle ultime riflessioni sulla comune russa. En S. Petrucciani (Ed.), Il pensiero di Karl Marx. Filosofia, politica, economia (pp. 73-114). Roma: Carocci.

Bobbio, N. (1976). Quale socialismo? Discussione di un'alternativa. Torino: Einaudi.

Bongiovanni, B. (1989). Le repliche della storia. Karl Marx fra la Rivoluzione francese e la critica della politica. Torino: Bollati Boringhieri.

Bourdieu, P. (2012). Sur l'État. Cours au Collège de France (1989-1992), Paris: Seuil. 
Engels, F. (2008). Über die politiche Aktion der Arbeiterklasse (1871). En MEW, 17 (1964, pp. 412-415), trad. it., Sullazione politica della classe operaia. En O, XXII (pp. 397-398). Napoli: La Città del Sole-Editori Riuniti.

Faber, K.-G., Ilting, K.-H. \& Meier, Ch. (1982). Macht-Gewalt. En O. Brunner, W. Conze \& R. Koselleck (eds.). Geschichtliche Grundbegriffe. Historisches Lexikon zur politisch-sozialen Sprache in Deutschland, 3 (pp. 817-935). Stuttgart: Klett-Cotta.

Gilbert, A. (1981). Marx's Politics. Communists and Citizens. New Brunswick: Rutger University Press.

Harvey, D. (2010). The Enigma of Capital and the Crises of Capitalism. Oxford: Oxford University Press.

Hobsbawm, E.J. (1978). Gli aspetti politici della transizione dal capitalismo al socialismo. En VV.AA., Storia del marxismo, Volume primo: Il marxismo ai tempi di Marx (pp. 245-287). Trad. it. Torino: Einaudi.

Koselleck, R. (1979). Vergangene Zukunft. Zur Semantik geschichtlicher Zeiten. Frankfurt a.M.: Suhrkamp.

Lefort, C. (2005). Le forme della storia. Saggi di antropologia politica (1978). Trad. it. Bologna: il Ponte.

Luporini, C. (1978). Critica della politica e critica delleconomia politica in Marx. Critica marxista, 1, 17-50.

Maguire, J. M. (1978). Marx's Theory of Politics. Cambridge: Cambridge University Press. Marx, K. (1975), Marx an Paul und Laura Lafargue (19/04/1870). En MEW, 32 (1965).

Marx, K. (1989). Das Kapital. Kritik der politischen Ökonomie. Dritter Band. Buch III: Der Gesamtprozeß der kapitalistischen Produktion (1894). En MEW, 25 (1964).

Marx, K. (1991). Das Kapital. Kritik der politischen Ökonomie. Erster Band. Buch I: Der Produktionsprozeß des Kapitals (1867, 1990). En MEW, 23 (1962).

Negri, A. (2006). I libri del rogo. Crisi dello Stato-piano, Partito operaio contro il lavoro, Proletari e Stato, Per la critica della costituzione materiale, Il dominio e il sabotaggio (1974-1977). Roma: DeriveApprodi.

Poulantzsas, N. (1964). L'examen marxiste de l'état et du droit actuels et la question de "l'alternative". Les Temps Modernes, 219-220, 274-302.

Sassen, S. (1998). Globalization and its Discontents. Essays on the New Mobility of People and Money. New York: New Press.

Sassen, S. (2006). Territory, Authority, Rights: From Medieval to Global Assemblages. Princeton: Princeton University Press. 
Sennett, R. (1998). The Corrosion of Character, The Personal Consequences of Work in the New Capitalism, New York: Norton.

Texier, J. (1998). Révolution et démocratie chez Marx et Engels. Paris: Puf.

Thompson, E. P. (1963). The Making of the English Working Class, Harmondsworth: Penguin.

Tronti, M. (1980). Operai e capitale (1966). Torino: Einaudi.

Tronti, M. (2008). Classe. En AA.VV., Lessico marxiano (pp. 65-76). Roma: il manifestolibri.

Vincent, J.-M. (2001). Un autre Marx. Après les marxismes. Lausanne: Éditions Page Deux. 
